
\title{
28 Research Suare \\ Prevalence of Subclavian Artery Stenosis in Patients With Coronary Artery Disease
}

Assem AbdelAziz Hashad ( $\nabla$ assemhashad@hotmail.com )

Department of Cardiovascular medicine, Cairo University https://orcid.org/0000-0001-8337-6481

Abdalla Elagha

Cairo University

Research

Keywords: Coronary Artery Disease, Subclavian stenosis, Color Doppler Ultrasound

Posted Date: August 24th, 2020

DOI: https://doi.org/10.21203/rs.3.rs-56393/v1

License: (c) (i) This work is licensed under a Creative Commons Attribution 4.0 International License. Read Full License 


\section{Abstract}

Background: Atherosclerosis is the most common and serious vascular disease that affects both the brain and the heart . (1) Subclavian stenosis/occlusion is a marker for atherosclerotic disease (eg, carotid, coronary \& lower extremity arteries) and future adverse cardiovascular events. ${ }^{(2)}$

In this study, we identified the prevalence of subclavian artery stenosis in patients presented with coronary artery disease (CAD) through changes in the Doppler tracing of vertebral arteries that appear to represent a clue of subclavian artery stenosis.

Methods. On the basis of extracranial Doppler ultrasound \& supplementary intracranial Doppler ultrasound, we assessed the pattern of Doppler waveform in both carotid \& vertebrobasilar systems among 100 consecutive patients who were hospitalized for CAD in the cardiovascular department.

Results. Among 100 consecutive CAD patients studied, we identified stenosis and occlusion of subclavian artery in 5 patients (5\%). In those patients, subclavian arterial disease was indirectly discovered by changes in Doppler waveforms of vertebral artery.

Conclusions. Prevalence of subclavian artery stenosis in patients with CAD is $5 \%$. Changes in the pulse contour of antegrade vertebral artery Doppler waveforms seem to represent a good screening method for subclavian steal phenomena.

\section{Introduction}

Atherosclerosis is the most common and serious vascular disease that affects both the brain and the heart. ${ }^{(1)}$ Subclavian stenosis/occlusion is a marker for atherosclerotic disease (eg, carotid, coronary \& lower extremity arteries) and future adverse cardiovascular events. ${ }^{(2)}$

Subclavian artery occlusion or a hemodynamically significant stenosis proximal to the origin of the vertebral artery results in lower pressure in the distal subclavian artery. As a result, blood flows from the contralateral vertebral artery to the basilar artery, and may flow in a retrograde direction down the ipsilateral vertebral artery, away from the brainstem with a subsequent deleterious neurologic effects \& significant morbidity as it can lead to symptomatic ischemic issues affecting the upper extremities \& brain. ${ }^{(3,485)}$

The incidence of subclavian stenosis in the general population ranges from $3 \%$ to $4 \%$. ${ }^{(2)}$ In this study, we identified the prevalance of subclavian stenosis in CAD patients by color Doppler evaluation of vertebral arteries that appear to represent a clue of subclavian artery stenosis .

\section{Subjects And Methods}


The present study was performed at Cairo University Hospital in Egypt. The protocol was approved by the local ethical committee of the Cairo University.

The study was performed for 100 consecutive patients who were hospitalized with CAD and underwent coronary angiography. All patients were subjected to:

- Clinical \& laboratory evaluation of cardiovascular risk factors

- Echocardiographic assessment of left ventricular systolic function was done in Ultrasound Laboratory in the Cardiovascular Department, Cairo University using Phillips HDI 5000 ultrasound equipment, Using : 2-4 $\mathrm{MHz}$ phased-array transducer

- Color Doppler Ultrasonographic evaluation of extra and intra cranial vasculature : a high frequency $(7-10 \mathrm{MHz})$ linear array transducer was employed to scan the carotid from the most proximal common carotid artery to the internal carotid artery as far as the mandible permitted. The identified parameters and measures named: Intima Media Thickness, presence of carotid plaque, degree of carotid stenosis and occlusion. The measures and quantifications of extra-cranial carotid atherosclerosis was performed according to the internationally published data. ${ }^{(6)}$ a $2-4 \mathrm{MHz}$ phased-array transducer through the following bone windows:

A- Temporal window to assess both right and left middle cerebral arteries, anterior cerebral arteries , posterior cerebral arteries as well as the intracranial part of internal carotid arteries .

B-Sub occipital window to assess the basilar artery and intracranial right and left vertebral arteries through the foramen magnum. Diagnosis of intracranial stenosis interpreted according to the internationally published criteria. ${ }^{(7)}$

- Evaluation of CAD severity of studied patients : Results of coronary angiography of studied patients were reviewed and severity of the CAD was assessed as follows: The diseased coronary vessels divided into 4 groups according to number of diseased vessels involvement : no significant vessel involvement , 1 significant vessel involvement, 2 significant vessel involvement, $\geq 3$ significant (multi) vessel involvement), ${ }^{(8)}$

\section{Statistical Analysis}

Descriptive statistics reported the mean age, gender distribution, prevalence or means for the atherosclerotic risk factors (hypertension, diabetes mellitus, dyslipidaemia, and smoking) \& for subclavian artery stenosis. Data was expressed as percent for discrete variables and as mean value +SD for continuous variables. Correlations between normally distributed variables were done using Pearson correlation coefficient. $\mathrm{P}$ value $\leq 0.05$ was considered significant for all tests.

\section{Results}


Baseline characteristics of all studied patients are given in table 1 . Their mean age of $56( \pm 7.8 \%) .93$ patients $(93 \%)$ were males \& 7 patients $(7 \%)$ were females.

Among patients studied: 43 patients (43\%) were smokers, 67 patients (67\%) were hypertensives, 52 patients (52\%) were diabetic and 52 patients (52\%) were dyslipidemic.

The patients had a variety of clinical presentations as follows; 46 patients (46\%) presented with unstable angina, 15 patients (15\%) presented with non ST elevation myocardial infarction (NSTEMI), 25 patients (25\%) presented with ST elevation myocardial infarction (STEMI) and 14 patients (14\%) presented with heart failure

Among patients studied, 5 patients were identified to had subclavian arterial disease. Their characteristic features \& comparison to patients without subclavian arterial disease are shown in table 2.

From table 2 ; The patients had subclavian arterial disease represent $5 \%$ of studied group. Their mean age of

51 (+6.2). All of them were males \& had no intracranial vascular stenosis. They were found to had statistically higher incidence of dyslipidemia, smoking, history of recent or old Stroke/ transient ischemic attack (TIA) , extra cranial moderate or severe carotid stenosis \& multi-vessel or left main coronary artery disease.

In patients having subclavian stenosis or total occlusion we observed a variety of Doppler waveforms in epsilateral vertebral artery that include:

1 - Retrograde flow: the whole Doppler wave form is below the baseline.

2 - Antegrade Doppler flow with a:

A - Transient sharp decline in blood flow velocity at mid systole, rounding of a subsequent second systolic peak, and restoration of forward flow in diastole.

B - Nadir of mid systolic cleft in blood flow velocity is at or below baseline, but with a rapid recovery of forward flow before diastole.

3- Retrograde \& antegrade Doppler flow with a : nadir of mid systolic cleft falls well below baseline signifying greater reversal of flow during systole. Forward flow is restored in diastole.

\section{Table 1. Clinical features of patients enrolled in the study}




\begin{tabular}{|ll|}
\hline Clinical features & Number of patients \\
\hline Age (year) & $56(+7.8 \%)$ \\
\hline Male gender & $93(93 \%)$ \\
\hline Smoking & $43(43 \%)$ \\
\hline Hypertension & $67(67 \%)$ \\
\hline Diabetes Mellitus & $52(52 \%)$ \\
\hline Dyslipidemia & $52(52 \%)$ \\
\hline Unstable angina & $46(46 \%)$ \\
\hline NSTEMI & $15(15 \%)$ \\
\hline STEMI & $25(25 \%)$ \\
\hline Heart failure & $14(14 \%)$ \\
\hline
\end{tabular}

Table 2. Clinical, echocardiographic and angiographic features in patients with Subclavian stenosis compared to patients without Subclavian stenosis 


\begin{tabular}{|c|c|c|c|}
\hline Clinical featuers & Subgroup A & Subgroup B & $P$ value \\
\hline Number of patients & 5 & 95 & \\
\hline Age (year) & $51( \pm 6.2)$ & $59( \pm 3.4)$ & NS \\
\hline Male gender & $5(100 \%)$ & 87 pts $(91.6 \%)$ & NS \\
\hline Smoking & 4 pts $(80 \%)$ & 39 pts $(41 \%)$ & $P<0.05$ \\
\hline Hypertension & 3 pts $(60 \%)$ & 64 pts $(67.4 \%)$ & NS \\
\hline Diabetes Mellitus & 3 pts $(60 \%)$ & 49 pts $(51.6 \%)$ & NS \\
\hline Dyslipidemia & 4 pts $(80 \%)$ & 48 pts $(50.5 \%)$ & $P<0.05$ \\
\hline Unstable angina & $2(40 \%)$ & $44(51.6 \%)$ & NS \\
\hline NSTEMI & $1(20 \%)$ & $14(14.7 \%)$ & NS \\
\hline STEMI & $1(20 \%)$ & $24(25.3 \%)$ & NS \\
\hline LV systolic dysfunction & $1(20 \%)$ & $13(13.7 \%)$ & NS \\
\hline Recent or old Stroke/TIA & $4(80 \%)$ & $38(40 \%)$ & $P<0.05$ \\
\hline Extra cranial carotid stenosis (moderate or severe) & $3(60 \%)$ & $13(13.7 \%)$ & $P<0.05$ \\
\hline Intracranial vascular stenosis & - & $6(6.3 \%)$ & $P<0.05$ \\
\hline Multi-vessel or left main coronary disease & $3(60 \%)$ & $30(31.6 \%)$ & $P<0.05$ \\
\hline
\end{tabular}

\section{Discussion}

- The current study describes the prevalence of subclavian steno-occlusive disease among patients with coronary artery disease ; $5 \%$ which is higher than in general population (3-4\%). The prevalence of subclavian artery stenosis in our duplex based study was nearly the same as that mentioned in trials that use a clinical criteria for subclavian artery stenosis. ${ }^{(9)}$ The incidence was $6 \%$. Diagnosis of subclvian artery stenosis in theses studies was diagnosed clinically as an interarm pressure difference of $\geq 15 \mathrm{mmHg}$.

We found a significant association between subclavian steno-occlusive disease and history of recent or old Stroke/ TIA , extra cranial moderate or severe carotid stenosis and multi-vessel or left main coronary disease. This association highlights the value for screening for subclavian stenosis or occlusion among ischemic heart disease patients at high risk or undergoing coronary artery bypass graft (CABG) operation.

Presence of subclavian artery stenosis may have an important impact in patients having CAD. The left internal mammary artery (LIMA) usually has an origin separate from the first part of the subclavian artery. 
Putting in mind that the LIMA is the first choice in coronary artery bypass grafting because of reduced cardiac events and superior graft patency $(10,11)$ \& that left subclavian artery is four times more likely to had stenosis than the right subclavian or innominate arteries. $(12,13,14)$ So, ischemic sequel of subclavian artery stenosis may extends to affect the heart in patients underwent or will do CABG operation.

This was observed in coronary-subclavian steal phenomenon in which the flow through the internal mammary artery may reverse and "steal" flow from the coronary circulation during upper extremity exercise. Identification of a significant subclavian artery stenosis prior to CABG can prevent this important problem. Those patients with a high-grade subclavian artery stenosis should be treated (percutaneously or surgically) prior to CABG operation. ${ }^{(15)}$

- Observation of vertebral artery Doppler waveform in our study revealed different blood flow patterns . These changes may reflect the different degree of severity of subclavian artery stenosis as mentioned with prior studies. ${ }^{(16-20)}$

The earliest manifestation of the subclavian steal physiology is a transient sharp deceleration of blood flow after the first systolic peak. The flow deceleration produces a notch in the pulse contour and gives rise to two systolic peaks: the first sharp, the second blunt and rounded. As the subclavian pathology progresses more, the systolic notch becomes more pronounced and the second systolic peak diminishes and broadens. The nadir of the notch becomes progressively lower until it reaches and eventually crosses the baseline. The reversal of flow during systole is at first minimal and transient but becomes increasingly more substantial until complete reversal of flow throughout the cardiac cycle is seen. ${ }^{(21)}$ The physiologic explanation for these Doppler findings may be twofold: a decrease in pressure in which blood flow velocity abruptly increases, and a loss of energy in which $78 \%$ disturbed or turbulent flow is present. ${ }^{(22)}$

- Our study had some limitations:

1 - It's not a population based study rather than hospital based study.

2 -We didn't reevaluate all patients who had negative duplex examination by another imaging modality to evaluate positive \& negative predictive values.

3 - Color Doppler ultrasound is an operator \& machine dependent. Therefore one would need very highly trained operator using high end machine.

4 - Some types of vertebral artery waveforms that denote subclavian artery stenosis are sensitive \& not specific (may occur in patients with no subclavian artery stenosis) . 
5 - Our study precludes mild \& moderate subclavian disease that are usually not associated with ipsilateral

vertebral artery Doppler waveform changes.

\section{Conclusions}

Prevalence of subclavian artery stenosis in patients with coronary artery disease is $5 \%$. Screening of CAD patients for subclavian artery stenosis may be important specially for those who will undergo CABG operation.

Changes in the pulse contour of antegrade vertebral artery Doppler waveforms seem to represent a good screening method for subclavian steal phenomena.

\section{Abbreviations}

\begin{tabular}{|ll|}
\hline CAD & Coronary artery disease \\
\hline NSTEMI & Non ST elevation myocardial infarction \\
\hline STEMI & ST elevation myocardial infarction \\
\hline TIA & Transient ischemic attack \\
\hline CABG & Coronary artery bypass graft operation \\
\hline LIMA & Internal mammary artery \\
\hline
\end{tabular}

\section{Declarations}

- Ethics approval and consent to participate: CU-2018/122. Consent obtained for all cases.

- Consent for publication: Obtained for all cases

- Availability of data and material: All data generated or analysed during this study are included in this published article [and its supplementary information files]

- Competing interests: The authors declare that they have no competing interests

- Funding: Not applicable

- Authors' contributions: AH analyzed and interpreted the patient data and was a major contributor in writing the manuscript. AE analyzed and interpreted the patient data and was a major contributor in writing the manuscript. All authors read and approved the final manuscript.

- Acknowledgements: Not applicable

\section{References}


1. Reddy KS, et al : Emerging epidemic of cardiovascular disease in developing countries. Circulation 1998; 97:596-601.

2. Victor M Ochoa and Yerem Yeghiazarians : Subclavian artery stenosis: A review for the vascular medicine practitioner Vascular Medicine 16(1) 29-34 @ The Author(s) 2010

3. Fisher, CM. A new vascular syndrome: "The subclavian steal." N Engl J Med 1961; 265:912.

4. Patel A, Toole JF. Subclavian steal syndrome - Reversal of cephalic blood flow. Medicine (Baltimore) $1965 ; 44: 289$.

5. Toole JF, McGraw CP. The steal syndromes. Annu Rev Med 1975; 26:321.

6. Andrei V. Alexandrov, Cerebrovascular Ultrasound in Stroke Prevention and Treatment, 2004

7. Ka Sing Wong, et al : Use of Transcranial Doppler Ultrasound to Predict Outcome in Patients With Intracranial Large-Artery Occlusive Disease Stroke. 2000;31:2641-2647

8. Ringqvist I, Fisher LD, Mock MI, et al. Prognostic value of angiographic indices of coronary artery disease from the Coronary Artery Surgery Study (CASS). The Journal of Clinical Investigation. 1983; 71(6): 1854-66.

9. Ramin Shadman, BA,* Michael H. Criqui, MD, MPH,et al : Subclavian Artery Stenosis: Prevalence, Risk Factors, and Association With Cardiovascular Diseases . Journal of the American College of Cardiology Vol. 44, No. 3, 2004

10. Loop FD, Lytle BW, Cosgrove DM, et al. Influence of the internal-mammary-artery graft on 10-year survival and other cardiac events. N Engl J Med 1986; 314:1-6.

11. Fiore AC, Naunheim KS, Dean P, et al. Results of internal thoracic artery grafting over 15 years: single versus double grafts. Ann Thorac Surg 1990; 49:202-208.

12. Rodriguez-Lopez JA, Werner A, Martinez R, Torruella LJ,

13. Ray LI, Diethrich EB. Stenting for atherosclerotic occlusive disease of the subclavian artery. Ann Vasc Surg 1999; 13: 254-260

14. Schillinger $M$, Haumer $M$, Schillinger $S$, Mlekusch $W$, Ahmedi R. Outcome of conservative versus interventional treatment of subclavian artery stenosis. J Endovasc Ther 2002; 9: 139-146

15. Hwang HY, Kim JH, Lee W, et al. Left subclavian artery stenosis in coronary artery bypass: prevalence and revascularization strategies. Ann Thorac Surg 2010; 89:1146.

16. Ringelstein EB, Zeumer H. Delayed reversal of vertebral artery blood flow following percutaneous transluminal angioplasty for subclavian steal syndrome. Neuroradiology 1984;26:189-198

17. Brancheareu A, Magnan PE, Espinoze H, Bartoli JM. Subclavian artery stenosis: hemodynamic aspects and surgical outcome. J Cardiovasc Surg (Torino) 1991;32:604-612

18. Yip PK, Liu HM, Hwang BS, Chen RC. Subclavian steal phenomenon: a correlation between duplex sonographic and angiographic findings. Neuroradiology 1992;34:279-282

19. Thomassen L, Aarli JA. Subclavian steal phenomenon: clinical and hemodynamic aspects. Acta Neurol Scand 1994;90:241-244 
20. Kotval PS, Babu SC, Shah PM. Doppler diagnosis of partial vertebral/subclavian steals convertible to full steals with physiologic maneuvers. J Ultrasound Med 1990;9:207-213

21. Mark A. Kliewer1, Barbara S. Hertzberg1, et al : Vertebral Artery Doppler Waveform Changes Indicating Subclavian Steal Physiology Department of Radiology, Duke University Medical Center, Received June 9, 1999; accepted after revision August 12, 1999.

22. Burns PN. Hemodynamics. In: Taylor KJW, Burns PN, Wells PNT, eds. Clinical applications of Doppler ultrasound. New York: Raven, 1988:56-61

\section{Figures}

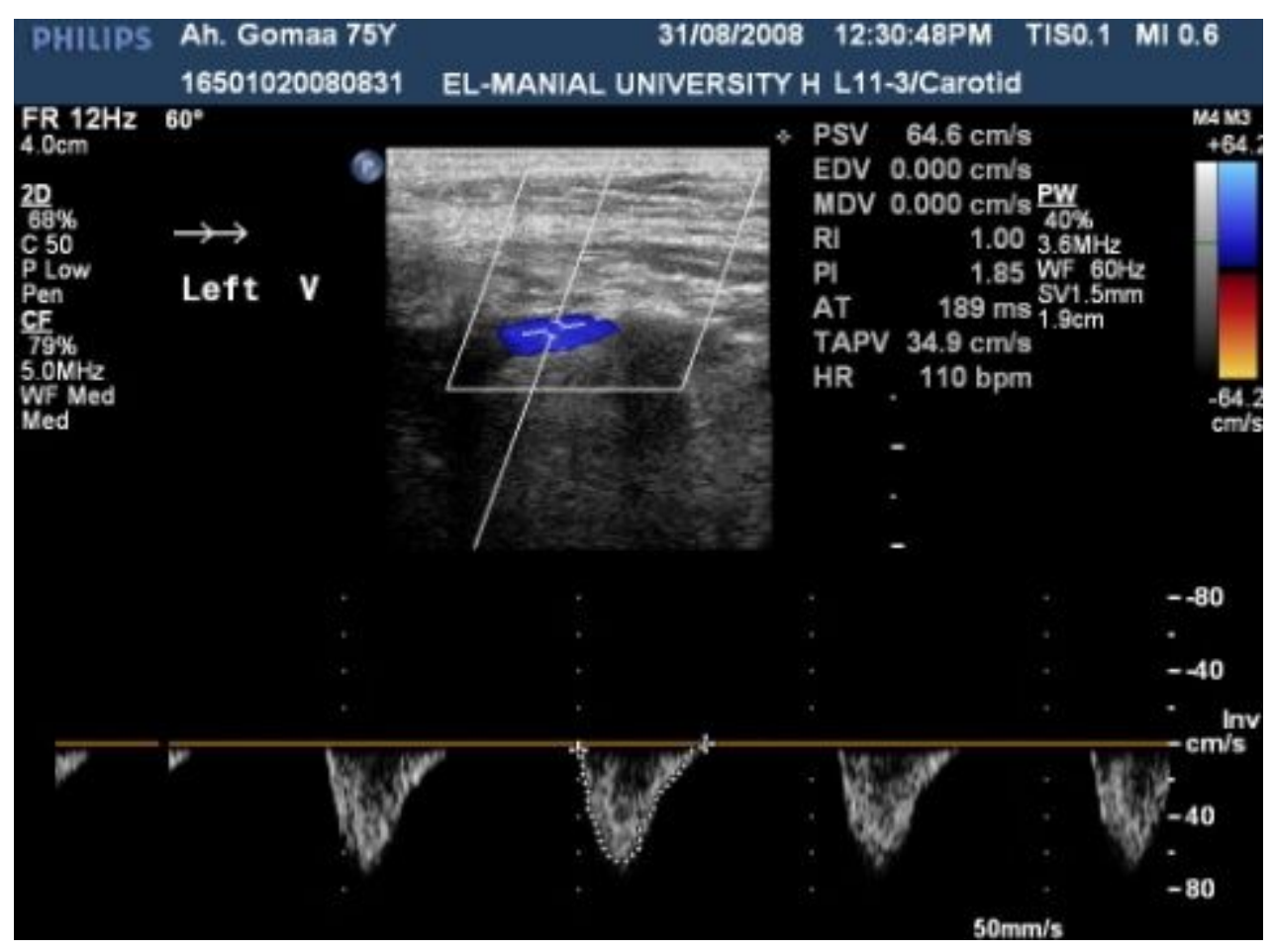

\section{Figure 1}

Color \& continuous wave Doppler Ultrasound of vertebral artery with an epsilateral subclavian artery total occlusion. It shows a totally retrograde Doppler waveform 

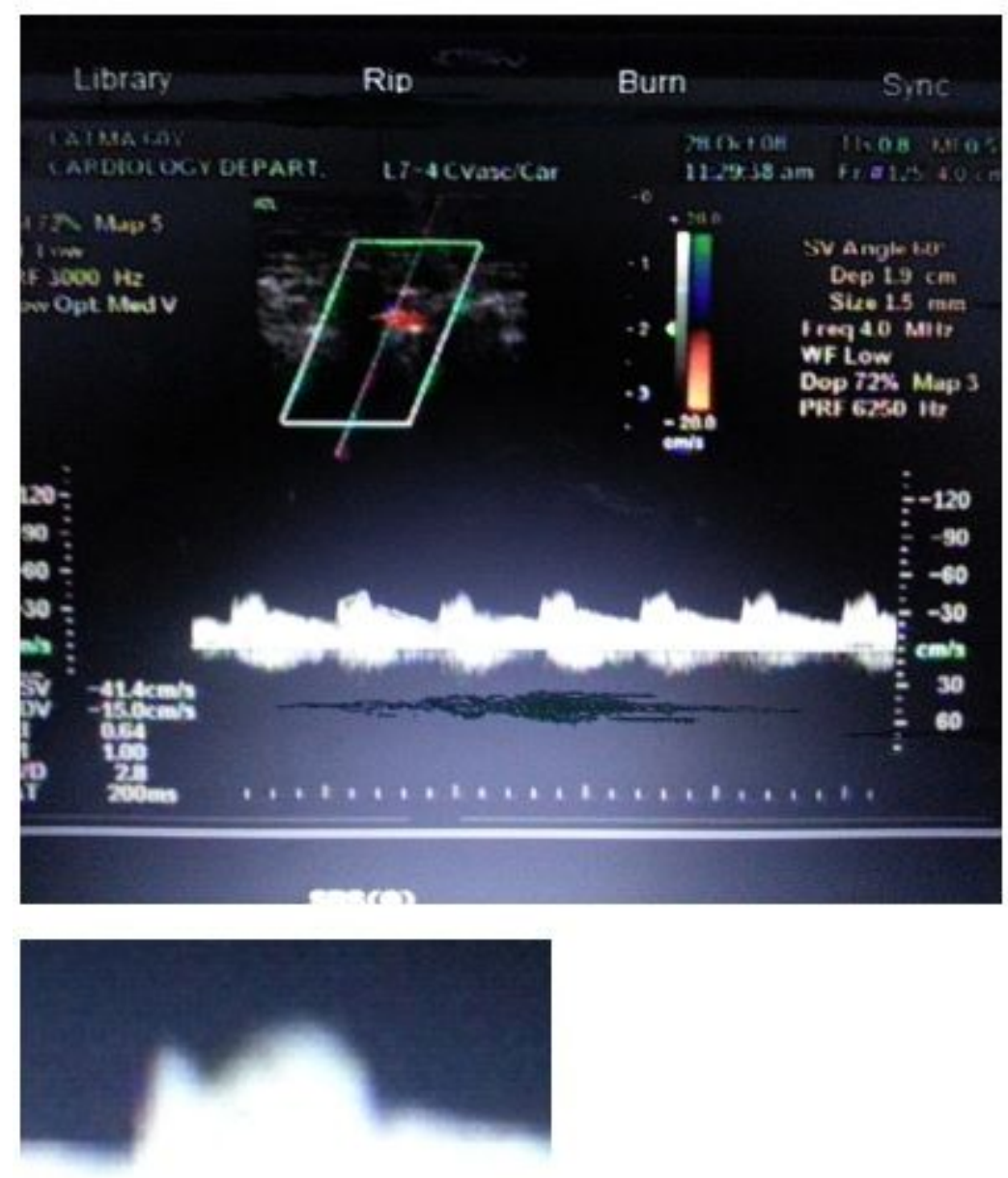

\section{Figure 2}

Color \& continuous wave Doppler Ultrasound of vertebral artery with a non significant stenosis of the epsilateral subclavian artery. It shows an antegrade flow with a : transient sharp decline in blood flow velocity at mid systole, rounding of a subsequent second systolic peak, and restoration of forward flow in diastole 


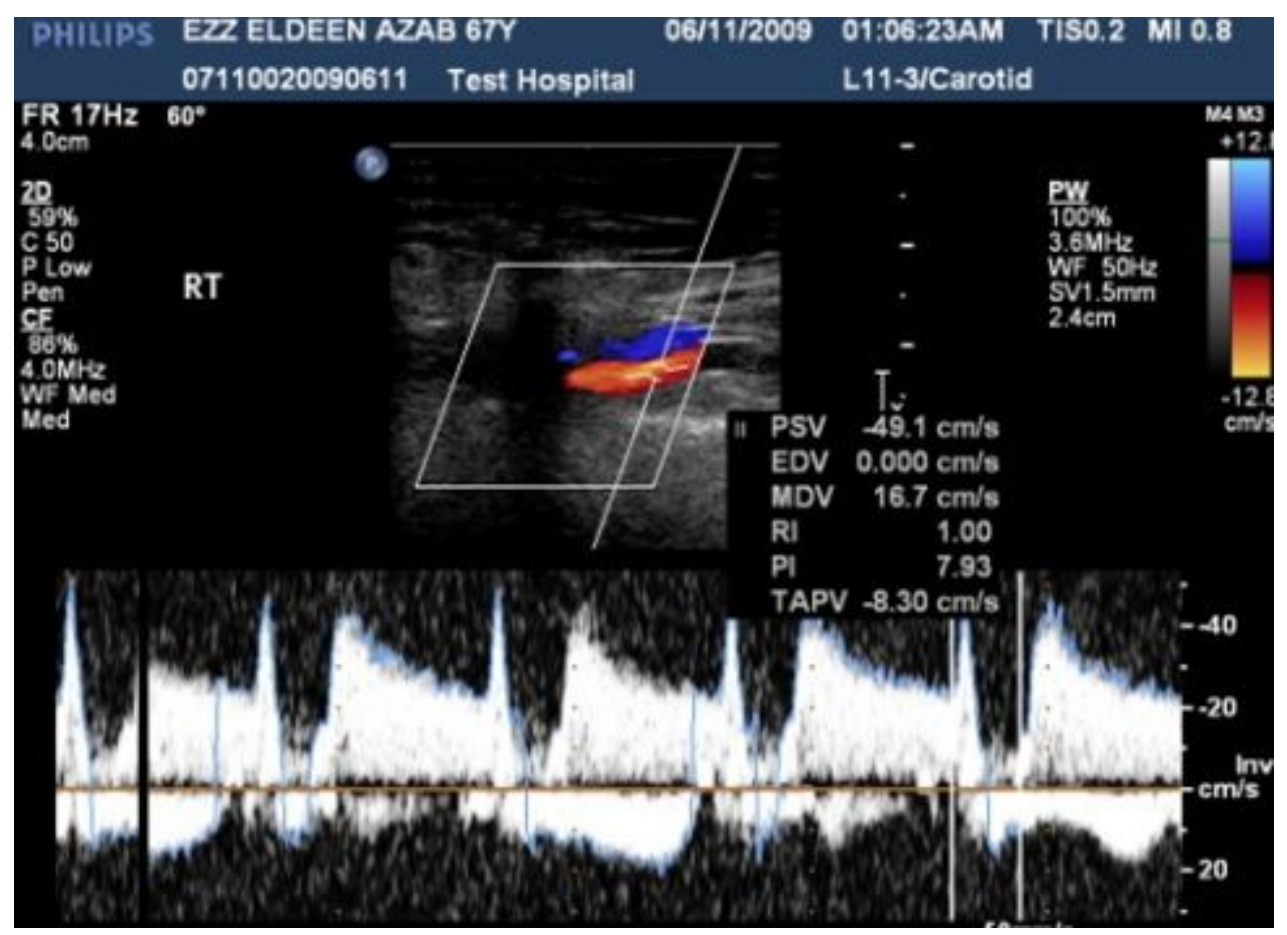

\section{Figure 3}

Color \& continuous wave Doppler Ultrasound of vertebral artery with a significant stenosis of the epsilateral subclavian artery . It shows an antegrade flow with a : nadir of mid systolic cleft in blood flow velocity is at or below baseline, but with a rapid recovery of forward flow before diastole

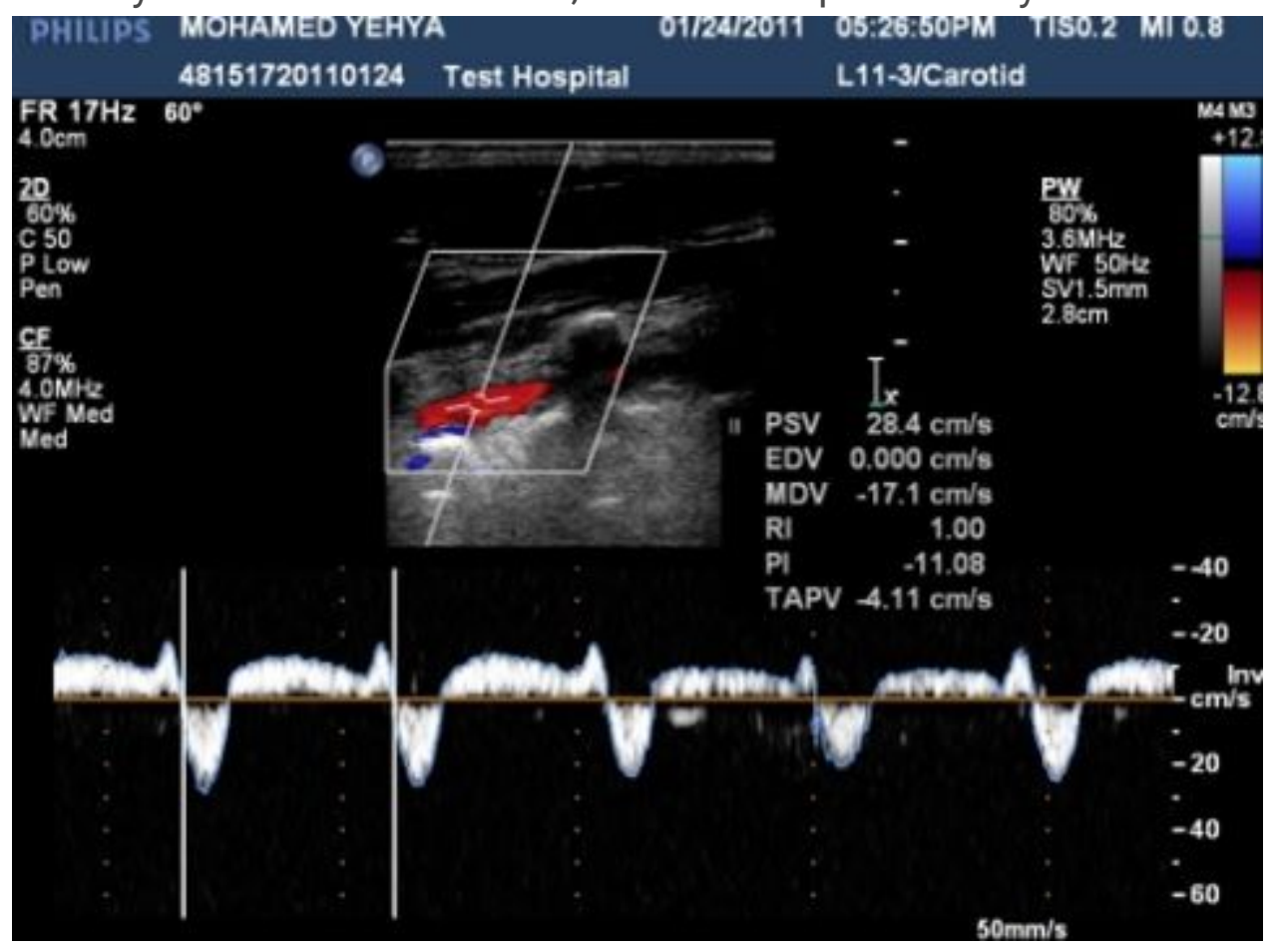

\section{Figure 4}

Color \& continuous wave Doppler Ultrasound of vertebral artery with a significant stenosis of the epsilateral subclavian artery with a : nadir of mid systolic cleft falls well below baseline signifying greater 
reversal of flow during systole. Forward flow is restored in diastole

\section{Supplementary Files}

This is a list of supplementary files associated with this preprint. Click to download.

- Supplementarymaterial.docx 